\title{
DISCURSO DE GERENTES SOBRE BARREIRAS DE ACESSO AO TESTE RÁPIDO ANTI-HIV NA ATENÇÃO PRIMÁRIA
}

\author{
MANAGERS' DISCOURSE ON BARRIERS \\ TO ACCESS HIV RAPID TEST IN \\ PRIMARY CARE
}

\section{DISCURSO DE LOS GERENTES SOBRE LAS BARRERAS AL ACCESO A LAS PRUEBAS RÁPIDAS DE VIH EN ATENCIÓN PRIMARIA}

\author{
Haline Costa dos Santos Guedes ${ }^{1}$ \\ José Nildo de Barros Silva Júnior ${ }^{2}$ \\ Amanda Haissa Barros Henriques ${ }^{3}$ \\ Débora Raquel Soares Guedes Trigueiro ${ }^{4}$ \\ Jordana Almeida Nogueira ${ }^{5}$ \\ Anne Jaquelyne Roque Barrêto ${ }^{6}$
}

Como citar este artigo: Guedes HCS, Silva Júnior JNB, Henriques AHB, Trigueiro DRSG, Nogueira JA, Barrêto AJR. Discurso de gerentes sobre barreiras de acesso ao teste rápido anti-HIV na atenção primária. Rev baiana enferm. 2021;35:e43561.

Objetivo: analisar as barreiras de acesso ao teste rápido anti-HIV na perspectiva do discurso de gerentes dos serviços de Atenção Primária à Saúde. Método: pesquisa exploratória, qualitativa, realizada em um distrito sanitário de João Pessoa, Paraíba, Brasil. A coleta de dados foi realizada em setembro de 2017 com 13 gerentes de saúde. O material empírico foi fundamentado no aporte teórico-metodológico da Análise do Discurso de matriz francesa. Resultados: identificou-se o bloco discursivo "Barreiras de acesso do usuário no sistema de saúde para realização do teste rápido anti-HIV na Atenção Primária à Saúde". Os discursos revelaram barreiras de acesso atreladas às dimensões: acessibilidade geográfica, disponibilidade, viabilidade e aceitabilidade. Considerações finais: as unidades possuem potencial para fortalecer a realização do teste rápido anti-HIV, entretanto, necessitam de apoio político que visem estratégias para fortalecer a realização do teste rápido com escopo na redução das barreiras de acesso.

Descritores: HIV. Diagnóstico Precoce. Atenção Primária à Saúde. Acesso aos Serviços de Saúde. Pesquisa Qualitativa.

Objective: to analyze the barriers to access HIV rapid test from the perspective of the discourse of managers of Primary Health Care services. Method: exploratory, qualitative research conducted in a health district of João Pessoa, Paraiba, Brazil. Data collection was performed in September 2017 with 13 health managers. The empirical material was based

\footnotetext{
Enfermeira. Especialização em Urgência, Emergência e Unidade de Terapia Intensiva. Universidade Federal da Paraíba. João Pessoa, Paraíba, Brasil. halineguedesenf@hotmail.com. http://orcid.org/0000-0003-1892-4503.

2 Enfermeiro. Mestre em Enfermagem. Docente da Faculdade São Vicente de Paula. João Pessoa, Paraíba, Brasil. http://orcid.org/0000-000 I-9958-8462.

Enfermeira. Mestre em Enfermagem. Docente do Instituto Federal da Paraíba. João Pessoa, Paraíba, Brasil. http://orcid.org/0000-000 I-8735-225X

Enfermeira. Doutora em Enfermagem. Docente da Faculdade de Enfermagem Nova Esperança. João Pessoa, Paraíba, Brasil. http://orcid.org/0000-000 I-5649-8256. Enfermeira. Doutora em Enfermagem. Docente do Programa de Pós-graduação em Enfermagem da Universidade Federal da Paraíba. João Pessoa, Paraíba, Brasil. http://orcid.org/0000-0002-2673-0285.

6 Enfermeira. Doutora em Enfermagem. Docente do Programa de Pós-graduação em Enfermagem da Universidade Federal da Paraíba. João Pessoa, Paraíba, Brasil. http://orcid.org/0000-0002-6852-8480.
} 
on the theoretical-methodological contribution of the Discourse Analysis of the French matrix. Results: the discursive block "Barriers of user access in the health system to perform the HIV rapid test in Primary Health Care" was identified. The discourses revealed barriers of access tied to the dimensions: geographical accessibility, availability, feasibility and acceptability. Final considerations: the units have the potential to strengthen the performance of the HIV rapid test, however, they need political support aimed at strategies to strengthen the performance of the rapid test with scope to reduce access barriers.

Descriptors: HIV. Early Diagnosis. Primary Health Care. Access to Health Services. Qualitative Research.

Objetivo: analizar los obstáculos al acceso a las pruebas rápidas del VIH desde la perspectiva del discurso de los gerentes de los servicios de Atención Primaria de Salud. Método: investigación exploratoria y cualitativa llevada a cabo en un distrito de salud de João Pessoa, Paraíba, Brasil. La recopilación de datos se realizó en septiembre de 2017 con 13 gerentes de salud. El material empírico se basó en la contribución teórico-metodológica del Análisis del Discurso de la matriz francesa. Resultados: se identificó el bloque discursivo "Barreras de acceso de los usuarios en el sistema de salud para realizar la prueba rápida del VIH en Atención Primaria de Salud". Los discursos revelaron barreras de acceso vinculadas a las dimensiones: accesibilidad geográfica, disponibilidad, viabilidad $y$ aceptabilidad. Consideraciones finales: las unidades tienen el potencial de fortalecer el desempeño de la prueba rápida del VIH, sin embargo, necesitan apoyo político dirigido a estrategias para fortalecer el desempeño de la prueba rápida con margen para reducir las barreras de acceso.

Descriptores: VIH. Diagnóstico Precoz. Atención Primaria de Salud. Acceso a los Servicios de Salud. Investigación Cualitativa.

\section{Introdução}

A incidência do Vírus da Imunodeficiência Humana (HIV) destaca-se como um problema de saúde pública no Brasil e no mundo devido à sua característica pandêmica e seu potencial enorme em ocasionar morte, apesar de ser prevenível e tratável. É notória a ascensão de ações para seu enfrentamento, entretanto, nem todo usuário tem acesso equitativo, sinalizando diversas barreiras para adentrar ao serviço de saúde ${ }^{(1)}$.

Segundo o Joint United Nations Programme on HIV/Aids (UNAIDS), em 2018 havia 37,9 milhões de pessoas vivendo com o HIV (PVHIV) no mundo. Destes, cerca de 8,1 milhões desconheciam sua condição sorológica ${ }^{(2)}$. No Brasil, de 2007 até junho de 2019, foram registrados 300.496 casos de HIV. No estado da Paraíba, por sua vez, entre os anos de 2017 e 2018, foram diagnosticados 1.282 novos casos de HIV e até o mês de outubro de 2019 foram diagnosticados 470 novos $\operatorname{casos}^{(3)}$.

A datar do surgimento do vírus, o governo brasileiro vem implementando ações e serviços com o propósito de reduzir a taxa de incidência da infecção e evitar as possibilidades da transmissão, dentre eles, a realização do Teste Rápido
(TR) anti-HIV. Este dispositivo organizacional vem se destacando como importante estratégia potencializadora na identificação dos casos positivos, auxiliando no diagnóstico precoce, de forma a ser utilizado como dispositivo com referência global na assistência aos usuários ${ }^{(4)}$.

O Ministério da Saúde, por meio da Portaria no 29, de 17 de dezembro de 2013, deliberou a ampliação da oferta dessa tecnologia diagnóstica para os serviços de Atenção Primária à Saúde (APS), favorecendo diretamente o processo de disponibilidade do TR para a identificação sorológica precoce, instauração do tratamento e interrupção da cadeia epidemiológica ${ }^{(5)}$.

A realização do TR anti-HIV, no contexto da APS, tem sido vista como uma resposta imediata à demanda de casos de HIV, pois, além de favorecer a ampliação do acesso e a formação do vínculo entre profissional e usuário, propicia o fluxo organizado da rede de atenção no nível primário, preconizado como principal porta de entrada do Sistema Único de Saúde (SUS) e eixo ordenador. Essa inserção de oferta de diagnóstico expressou uma nova face das políticas de saúde, para consolidar o modelo descentralizado ${ }^{(6)}$. 
Destarte, é fundamental analisar as barreiras do acesso ao TR anti-HIV na APS para melhor compreensão do uso dos serviços de saúde e suas limitações, já que essas unidades devem fornecer acolhimento, diagnóstico, tratamento precoce, além de uma oferta que contemple os princípios da universalidade e acessibilidade. Tais prerrogativas sinalizam a necessidade de fornecimento adequado de testes para as unidades, ampliação da oferta do exame e expansão da capacitação para os profissionais da Equipe de Saúde da Família executarem o procedimento de forma correta ${ }^{(7)}$.

Após busca em bases de dados nacionais e internacionais, foi possível averiguar estudos que apontam que os profissionais da APS reconhecem a importância do TR anti-HIV como estratégia de cuidado, apesar de muitas vezes não ser realizado conforme preconizado, em função de fatores estruturais e operacionais ${ }^{(8-9)}$. Entretanto, quando se trata de pesquisas que contemplem o aspecto organizacional das dificuldades que permeiam o acesso ao TR anti-HIV na APS, não foram identificados estudos que versem sobre as barreiras de acesso indicadas no discurso de gerentes de saúde, o que prejudica a compreensão da integralidade do cuidado e a tomada de decisão no combate a esta infecção.

Tendo em vista a potencialidade da realização do TR anti-HIV enquanto estratégia de prevenção e promoção de saúde, evidencia-se a necessidade de realizar estudos que analisem a temática e, especificamente, as barreiras de acesso ao TR na APS, sinalizando as limitações e os desafios inseridos nesse contexto, com ênfase na perspectiva dos gestores de saúde, atores importantes para a efetivação e a operacionalização do acesso ao TR na prática, uma vez que o processo envolve desde a compra até a disponibilidade do TR nos serviços de APS ${ }^{(10)}$.

Essa análise permite subsidiar discussões com vistas a eventuais intervenções e adaptações que aprimorem o acesso ao TR anti-HIV no âmbito da APS, demonstrando a relevância desta investigação. No tocante à temática, o presente estudo parte da seguinte questão norteadora: O que revelam os discursos dos gerentes de saúde da APS acerca das barreiras de acesso ao TR anti-HIV?
Desse modo, este estudo objetivou analisar as barreiras de acesso ao TR anti-HIV na perspectiva do discurso de gerentes dos serviços de APS.

\section{Método}

Trata-se de pesquisa exploratória de natureza qualitativa, guiada pelos critérios incluídos no checklist do Consolidated Criteria for Reporting Qualitative Research (COREQ), fundamentada no aporte teórico-metodológico da Análise do Discurso (AD) de matriz francesa pecheutiana, que epistemologicamente se enlaça na linguística, na psicanálise e no materialismo histórico ${ }^{(11)}$.

A AD propõe analisar a linguagem como prática social, por meio de um sujeito inconsciente, sem emitir juízo à linguagem, como certo ou errado, mas expor o que é e como funciona, estando interessada em como será permitido o discurso. Tendo em vista que as práticas do pensamento são evidenciadas mediante os discursos, compete à $\mathrm{AD}$ francesa explicar o processo de construção da línguagem, posto que não há discurso sem sujeito e nem sujeito sem ideologia ${ }^{(11)}$.

O estudo foi realizado nos serviços de APS de um Distrito Sanitário na região leste de João Pessoa, capital da Paraíba. O local eleito para integrar o cenário de pesquisa foi selecionado por apresentar o maior quantitativo de unidades de saúde e usuários que acessam o TR anti-HIV. Os participantes foram selecionados de forma intencional, considerando-se como critério de inclusão ter atuação mínima de seis meses como gestor no sistema local de saúde. Foram excluídos os gerentes de saúde que estavam de férias ou em licença médica no período da coleta.

O Distrito Sanitário pesquisado possui 18 gerentes de saúde, entretanto, após contato com os participantes, 13 foram incluídos no estudo por estarem em conformidade com os critérios estabelecidos. O Programa Gerente Saúde em vigor no município é respaldado por norma estabelecida pelo Ministério da Saúde (MS) e a Organização Mundial da Saúde (OMS), inclusa na Política Nacional de Atenção Básica (PNAB), recebendo caráter de referência para todos os municípios brasileiros, possui posição de gestão 
e acompanhamento da Estratégia Saúde de Família (ESF) do município selecionado.

A coleta de dados ocorreu em setembro de 2017, por meio de técnica de entrevista com o aporte de roteiro semiestruturado, organizado de acordo com o objetivo da pesquisa, com questões abertas sobre a organização dos serviços de APS quanto ao acesso ao TR anti-HIV. As entrevistas foram agendadas previamente, conforme a disponibilidade do participante, e foram realizadas em ambiente privativo escolhido por cada profissional, de forma individual, após a assinatura do Termo de Consentimento Livre e Esclarecido (TCLE). As gravações foram feitas com auxílio de um smartphone, com duração média de 20 minutos. Posteriormente, as entrevistas foram transcritas para um diário de campo, o que favoreceu a organização do corpus da pesquisa. Com o intuito de preservar o anonimato, os sujeitos colaboradores foram identificados pela letra G, alusiva ao nome gerente, seguida de algarismos arábicos (G1 a G13).

Tendo em vista que a concepção da $\mathrm{AD}$ de linha francesa busca expressar o caráter histórico, voltado à centralidade no discurso e no realce da observação histórica com base em perspectivas divergentes, e não na procura do sentido verdadeiro, o dispositivo analítico segue dois momentos distintos e complementares: a análise em si e a escrita da análise ${ }^{(11)}$.

A primeira etapa, a análise em si, procede a circunscrição do conceito-análise, que possui como escopo da análise a saturação que é estabelecida pela ausência de novos elementos no discurso, a ponto de ser encerrado. Assim, o corpus discursivo foi definido mediante leitura flutuante, após leitura analítica, que possui a função de assistir o analista na compreensão dos sentidos referentes à resposta de três perguntas heurísticas: Qual é o conceito-análise presente no texto? Como o texto constrói o conceito-análise? A que discurso pertence o conceito-análise construído da forma que o texto constrói ${ }^{(11)}$ ?

Posteriormente, foram realizadas leituras exaustivas para possibilitar o reconhecimento dos sentidos e das marcas textuais até haver a saturação dos sentidos.

Em seguida, buscou-se identificar o sentido edificado pelo discurso dos gerentes de saúde em referência ao objeto de estudo, enfatizando o funcionamento da ideologia na textualização ${ }^{(11)}$. O conceito-análise estabelecido neste estudo foi "Barreiras de acesso ao TR anti-HIV". Esse conceito embasa-se nas marcas textuais denotadas no corpus discursivo elaborado por meio dos discursos dos sujeitos gerentes.

A segunda etapa, a escrita da análise, envolveu a caracterização da análise por meio da contextualização e elucidação da temática, e da explicação do dispositivo teórico-analítico ${ }^{(11)}$. Dessa forma, emergiu o seguinte bloco discursivo: Barreiras de acesso ao usuário no sistema de saúde para realização do TR anti-HIV na APS. Para a categorização analítica utilizou-se as dimensões de acesso: acessibilidade geográfica, disponibilidade, viabilidade e a aceitabilidade ${ }^{(12-13)}$.

Registra-se que foram respeitados os princípios éticos e legais estabelecidos na Resolução $n^{\circ} 466 / 2012$, do Conselho Nacional de Saúde, atinente a pesquisas que envolvem seres humanos. O projeto de pesquisa foi aprovado pelo Comitê de Ética em Pesquisa (CEP) das Faculdades de Enfermagem e Medicina Nova Esperança, sob Parecer no ${ }^{\underline{0}}$ 109/2017 e CAAE 72757817.6.0000.5179.

\section{Resultados}

O Quadro 1 contempla um corpus construído por meio das dimensões e das respectivas barreiras de acesso ao TR anti-HIV na APS com os fragmentos discursivos de 13 gerentes de saúde com tempo de trabalho superior a seis meses na APS, dentre os quais, 4 são assistentes sociais, 4 fisioterapeutas, 2 profissionais de educação física, 2 fonoaudiólogas e 1 administradora. Deste quatitativo apenas um era do sexo masculino e os demais, do feminino. Assim, estes discursos conduzirão a discussão do conceito análise estabelecido. 
Quadro 1 - Recorte discursivo dos gerentes de saúde relacionado às dimensões e suas barreiras correspondentes que distanciam os usuários do serviço para realização do TR anti-HIV na APS. João Pessoa, Paraíba, Brasil - 2017

\begin{tabular}{|c|c|c|}
\hline Dimensão & Barreiras & Fragmentos discursivos \\
\hline \multirow[t]{3}{*}{$\begin{array}{l}\text { Acessibilidade } \\
\text { Geográfica }\end{array}$} & $\begin{array}{l}\text { Infraestrutura } \\
\text { inadequada }\end{array}$ & $\begin{array}{l}\text { [...] aqui a unidade ela é pequena para a comunidade que a } \\
\text { gente está atendendo [...]. (G10). }\end{array}$ \\
\hline & $\begin{array}{l}\text { Localização do } \\
\text { serviço distante } \\
\text { dos usuários }\end{array}$ & $\begin{array}{l}\text { [...] daqui enquanto isolada [distante das residências dos } \\
\text { usuários] não, sempre quando tem teste rápido a gente faz } \\
\text { uma permuta [...]. (G4). }\end{array}$ \\
\hline & $\begin{array}{l}\text { Segurança local } \\
\text { vulnerável }\end{array}$ & $\begin{array}{l}\text { [...] a gente está atendendo três áreas descobertas, só áreas } \\
\text { extremamente de risco, são áreas de vulnerabilidade total, } \\
\text { tanto na pobreza, quanto no uso de drogas [...]. (G10). }\end{array}$ \\
\hline \multirow[t]{4}{*}{ Disponibilidade } & $\begin{array}{l}\text { Articulação } \\
\text { fragilizada entre } \\
\text { os pontos de } \\
\text { atenção }\end{array}$ & $\begin{array}{l}\text { Aqui não é ofertado [TR anti-HIV], se fosse a gente } \\
\text { encaminharia (G6). } \\
\text { [...] ele foi encaminhado para o Clementino [Hospital de } \\
\text { referência], onde faz toda essa parte psicológica, a parte dos } \\
\text { coquetéis é todo encaminhado pra lá [...] faz o exame, se deu } \\
\text { positivo a gente encaminha direto pra lá [...]. (G11). } \\
\text { Como aqui não tem [TR anti-HIV], se for identificado algum } \\
\text { usuário que tenha algum sintoma, referencia pro Clementino } \\
\text { [Hospital de referência] [...]. (G12). }\end{array}$ \\
\hline & $\begin{array}{l}\text { Horário de } \\
\text { funcionamento } \\
\text { centralizado }\end{array}$ & $\begin{array}{l}\text { [...] a gente informa e o usuário já vai saber que naquele dia } \\
\text { vai haver uma ação e que nesse dia da ação, vai haver a } \\
\text { oferta do teste rápido do HIV [...]. (G8). }\end{array}$ \\
\hline & $\begin{array}{l}\text { Falta de } \\
\text { familiaridade } \\
\text { com a } \\
\text { capacitação } \\
\text { profissional }\end{array}$ & $\begin{array}{l}\text { A dificuldade para o usuário é porque nós não temos os } \\
\text { profissionais com esse curso [...]. (G1). } \\
\text { [...] a dificuldade maior é que a gente não tem nenhum } \\
\text { profissional da equipe capacitado para realizar esses testes } \\
\text { [...]. (G7). }\end{array}$ \\
\hline & $\begin{array}{l}\text { Escassez } \\
\text { de insumo }\end{array}$ & $\begin{array}{l}\text { [...] de vez em quando acaba e demora para chegar, o repasse } \\
\text { demora a acontecer [...] ou seja, falta de insumo [...]. (G2). } \\
\text { O teste rápido é que as vezes é que falta, agora mesmo no } \\
\text { momento está faltando [...]. (G5). }\end{array}$ \\
\hline Viabilidade & $\begin{array}{l}\text { Geração de } \\
\text { custos evitáveis }\end{array}$ & $\begin{array}{l}\text { [...] que eles tenham que se deslocar para um local [Hospital } \\
\text { de referência] que necessita por exemplo: recurso financeiro, } \\
\text { de uma passagem de transporte, essa questão toda. (G3). }\end{array}$ \\
\hline \multirow[t]{2}{*}{ Aceitabilidade } & $\begin{array}{l}\text { Discriminação de } \\
\text { PVHIV }\end{array}$ & $\begin{array}{l}\text { [...] mas eu acredito que esse tipo de usuário é o Clementino } \\
\text { [Hospital de referência] para o tratamento [...]. (G13). }\end{array}$ \\
\hline & Estigmatização & $\begin{array}{l}\text { [...] agora quando chega aqui na ação noturna e a gente } \\
\text { diz tem teste rápido, é de quê? De Aids? A maioria não quer, } \\
\text { parece que tem até medo de saber se tem [...]. (G9). }\end{array}$ \\
\hline
\end{tabular}

Fonte: Elaboração própria.

\section{Discussão}

A AD de linha francesa foi utilizada como dispositivo teórico-metodológico para elucidar o decurso de construção por meio da línguagem e para interpretar o corpus deste estudo com base no conceito-análise "barreiras de acesso para a realização do TR anti-HIV", a qual atende as ações do pensamento encontradas nos discursos dos gerentes de saúde, na perspectiva do objeto deste estudo, revelando a condução da ideologia na textualização ${ }^{(11)}$.

Analisando-se os fragmentos discursivos e as marcas textuais, foi percebido situações que sinalizam barreiras para a realização do exame relacionadas às dimensões acessibilidade geográfica, disponibilidade, viabilidade e aceitabilidade, respectivamente, que conduzirão a discussão 
do conceito-análise estabelecido. Nos discursos foram reveladas circunstâncias que fragilizam o acesso e oportunizam barreiras que distanciam os usuários da realização do exame.

Os discursos dos gerentes de saúde remetem à dimensão acessibilidade geográfica, vinculada ao acesso ao serviço de saúde, e está relacionada à adequação da localização dos serviços em referência à localização dos usuários ${ }^{(12-13)}$.

As barreiras reveladas no discurso atrelada à "dimensão acessibilidade geográfica" foram: infraestrutura inadequada, localização do serviço distante dos usuários e segurança local vulnerável. O tamanho da estrutura física é visto como uma limitação ao acesso do TR, uma vez que para melhor assistir à demanda da comunidade no espaço necessitaria de uma unidade de atendimento maior.

Estudo sobre testagem anti-HIV na APS identificou diferenças na qualidade da assistência e acesso ao TR entre duas unidades com estruturas físicas dissemelhantes. A literatura aponta que um espaço menor influencia negativamente para o atendimento adequado na execução da testagem, enquanto a de maior espaço potencializa a adesão, por disponibilizar espaços para testagem, privacidade e ambiente para reuniões estratégicas e acolhimento $^{(14)}$.

Outro estudo realizado em um município da Bahia identificou que o espaço reservado e privativo é algo almejado pelo usuário para a realização do exame. A estrutura física adequada nas unidades da APS influenciam positivamente nas práticas dos profissionais de saúde, favorecendo a realização das intervenções com qualidade técnica e condições mais humanizadas de trabalho ${ }^{(15)}$.

Outra barreira identificada foi a localização do serviço distante dos usuários, citada nas segmentações de G4, que resgata em seu discurso a distância das casas dos usuários para chegar na unidade, além do deslocamento de suas residências para a unidade referenciada, devido ao local não ofertar o TR. A fala desse gestor caracteriza importante entrave no acesso ao TR anti-HIV, pois propicia o desencontro do usuário com o serviço referenciado, podendo os profissionais da unidade destinada possuírem outras demandas e acabar negligenciando a realização do exame.

Assim, a distância entre a localização geográfica dos serviços de saúde e a residência do usuário deve ser superada, para que não haja peregrinação dos pacientes e desencontro com o serviço entre os diferentes locais e municípios para o acesso ao $\mathrm{TR}^{(16)}$.

Findando a dimensão acessibilidade geográfica, G10 menciona os riscos relacionados à segurança no entorno da unidade, configurando-se como barreira de acesso ao TR anti-HIV. Além disso, ele menciona a vulnerabilidade social, inclusive quanto ao uso de drogas, de forma a fragilizar o serviço, e propiciando ao usuário optar por não ir à unidade da APS para a realização do TR, devido à insegurança local.

Estudo destaca a importância da segurança da APS na oferta do cuidado seguro e com qualidade; para isso, é necessário articular responsabilidades relacionadas à gestão, aos profissionais, à estrutura física e à segurança no entorno do serviço de saúde ${ }^{(17)}$, para que o paciente encontre na unidade a tranquilidade e a sensação de segurança necessárias para sentir-se acolhido naquele ambiente.

A "disponibilidade" é outra dimensão que emergiu nos discursos, estando atrelada com as barreiras articulação fragilizada entre os pontos de atenção, horário de funcionamento centralizado, falta de familiaridade com a capacitação profissional e escassez de insumo. Tal situação permite a reflexão na oferta de ações de saúde no lugar certo e na hora certa, a fim de assistir às reais necessidades da população, à capacidade do serviço em atender os usuários, além de aspectos quanto à marcação de consulta, integralidade da assistência, qualidade e longitudinalidade do cuidado ${ }^{(12-13)}$.

Os fragmentos discursivos dos enunciados de G6, G11 e G12 revelam sentidos que indicam barreiras para a realização do TR anti-HIV nos serviços de APS, por meio das marcas textuais como "encaminhar e referenciar". Tal discurso justifica-se como memória discursiva em alusão à ideologia biomédica e hospitalocêntrica 
materializada pela lembrança social estabelecida na historicidade, pois sinalizaram sentidos que buscam em seu interdiscurso a assistência curativa e especializada, ao direcioná-los para os Centros de Testagem e Aconselhamento (CTA) que funciona no Complexo Hospitalar Clementino Fraga, sem considerar que o CTA deve ter um caráter complementar à assistência ${ }^{(18)}$.

Estudo feito no Sul do país aborda a necessidade de encaminhar o usuário para o CTA, como ponto para construção de estratégias conjuntas de prevenção, favorecendo o fluxo de atendimento na rede de atenção de forma organizada, ordenada e contrarreferenciada, que objetive erradicar comportamentos de risco, que possam ser dispensados para complementação de uma assistência integral em distintos serviços de saúde ${ }^{(8)}$.

Os sentidos atribuídos no discurso de G8 remete à historicidade da assistência representada pelo modelo campanhista devido as ações de saúde serem intervenções pontuais, sem oferta de horário alternativo de atendimento conforme a necessidade do paciente, implicando em impasses na adesão do indivíduo à procura do TR.

Pesquisa desenvolvida na APS no Sul do país identificou que o modelo de agendamento é uma importante ferramenta para otimizar a qualidade do acesso ao TR na APS, o qual possibilita o atendimento em dias específicos para demanda espontânea e preserva os demais dias para a continuidade de rotina e promoção à saúde, de forma a absorver parte da população que não se adapta ao atendimento programado ${ }^{(19)}$.

Portanto, é possível observar estudos que atribuem algumas indagações a respeito da demanda espontânea em referência à limitação da procura, em que o usuário tendencia a buscar os serviços de assistência técnica já no processo de adoecimento com caráter curativo, contrapondo-se ao preconizado pelo MS, que visa à prevenção e promoção à saúde ${ }^{(20)}$.

Nas falas de G1 e G7 foram sinalizadas a necessidade de profissionais capacitados. Estudo aponta que é de competência multiprofissional a execução do TR anti-HIV; o MS exige apenas a graduação e a capacitação para efetuar o exame, destacando-se a importância do envolvimento no processo de todas as categorias, cuja ação potencializa a implementação do TR na $\operatorname{APS}^{(9)}$.

Estudo desenvolvido no Nordeste do país ressalta a necessidade de capacitar todos os profissionais que participam da equipe, para propiciar o fortalecimento da interação e a cooperação entre a equipe na partilha de conhecimentos na execução do $\mathrm{TR}^{(18)}$.

A política vigente de enfrentamento ao HIV estima que os serviços ofertados pela APS sejam resolutivos e acessíveis, pois a realização do TR protagoniza a entrada dos usuários no serviço, otimizando a oferta em saúde ${ }^{(16)}$, o que reforça a necessidade dos profissionais da APS, enquanto atores sociais, qualificarem-se para realizar o TR anti-HIV.

De modo a encerrar as barreiras de acesso atreladas à dimensão disponibilidade, os discursos de G2 e G5 mencionam marcas textuais referentes à "falta de insumo", a escassez de insumo e a demora de reposição como maior déficit de fragilidade para a realização do TR. A escassez desse dispositivo distancia os usuários da realização da testagem, fragilizando o seu acesso ao exame. Para potencializar a testagem na APS, faz-se necessária a logística de entrega e disponibilidade do insumo, isto é, do kit do TR anti-HIV no serviço de saúde ${ }^{(18)}$.

Com base na sequência discursiva, a dimensão "Viabilidade" tem como barreira de acesso a geração de custos evitáveis, presente no fragmento da fala de G3, que faz alusão ao grau de adequação entre o custo da utilização dos serviços de saúde e a capacidade de pagamento dos indivíduos ${ }^{(12-13)}$.

Tal barreira transmite a dificuldade do usuário para se deslocar para os serviços especializados, já que a responsabilidade do diagnóstico foi transferida da primeira instância, a APS, para a atenção terciária. A fala dos profissionais revelam que eles percebem a distância da moradia para o serviço de referência hospitalar como um dificultador, pois os usuários precisam gastar com transporte, a fim de acessar o TR.

É válido frisar que essa barreira é um achado do nosso estudo e há limitações de publicações 
que analisam esse componente, sendo encontrado apenas uma pesquisa que constatou a modalidade de deslocamento "a pé" como a principal forma de acessibilidade aos serviços da $\operatorname{ESF}^{(21)}$.

A última dimensão desta pesquisa trata-se da "aceitabilidade", que pode ser entendida como as ações dos profissionais de saúde na perspectiva das características dos usuários mutuamente, estando relacionada à reciprocidade do profissional-usuário e usuário-sistema de saúde ${ }^{(1,12)}$.

As barreiras identificadas nesta dimensão remetem à discriminação de pessoas vivendo com HIV e estigmatização. Seguindo a linha de interpretação dos sentidos foi evidenciado na marca textual de G13 "esse tipo de usuário" em apologia à discriminação por atribuir um rótulo ao usuário. Além disso, tal discurso materializa a recordação social instituída em atitudes que têm foco negativo em PVHIV.

A discriminação é resultado ideológico e prático do estigma construído pela sociedade, resultando em injustiças sociais, seja ela por omissão ou falta de ação, alicerçado em atitudes com escopo em estereótipos, na premissa de trazer desvantagens às pessoas rotuladas por estarem infectadas pelo $\mathrm{HIV}^{(22)}$.

Em estudo realizado na África do Sul foi visto que os profissionais, em seu âmbito laboral, ainda vinculam o HIV à injúria social e encontram uma maneira de se desresponsabilizar do usuário com escopo na testagem e medicalização, não estando familiarizados com o acolhimento holístico desse indivíduo ${ }^{(23)}$.

Em outro estudo também na África do Sul, usuários adolescentes relataram experiências discriminatórias, como exclusão, detratação, cuidados de saúde precários entre profissionais de saúde e público em geral. Além disso, o estudo aponta que a discriminação impulsiona os comportamentos de maior risco, distancia os usuários da testagem, iniciação e adesão ao tratamento medicamentoso, fragilizando o vínculo do usuário com o serviço ${ }^{(24)}$.

Por fim, a última barreira de acesso ao TR anti-HIV faz menção ao estigma social, em que G9 busca na memória discursiva a renovação da historicidade do estigma por meio da materialização de uma lembrança social pré-estabelecida, perpetuada ao longo do tempo, em que o indivíduo tinha que conviver com várias nomeações e adjetivações pejorativas $^{(22)}$.

A detecção do HIV traz consigo uma condenação e exclusão adotadas pela sociedade mediante a estigmatização, cuja materialização corrobora para tensões na realização do TR. Por isso, mesmo diante da ampliação da oferta do TR, o exame na APS é visto como um elemento que constrange, pela possibilidade do diagnóstico colocar o usuário em exposição da sua condição sorológica no ambiente da família e comunidade $^{(4)}$.

No Sul do Brasil, foi desenvolvido um estudo com discurso semelhante, em que uma das participantes mencionou que se sentia inibida e constrangida quando os profissionais falavam da oferta do TR. Assim, compreende-se que a realização do teste para detecção do HIV é permeada por outras demandas, referentes à privacidade e confidencialidade, além de relacionar a detecção do HIV a aspectos morais que favorecem o processo de estigmatização na sociedade ${ }^{(25)}$.

O estigma e a discriminação do HIV são caracterizados como importantes barreiras de acesso ao serviço na perspectiva da prevenção, tratamento e ações em todo o cenário da saúde. É primordial o apoio holístico para enfrentar essa infecção com adoção de políticas sociais de enfrentamento às desigualdades, além da potencialização do empoderamento das pessoas acometidas pelo vírus.

Apreende-se, portanto, que a realização do TR anti-HIV, no nível de APS, não converge com a acessibilidade do usuário à testagem, na proporção em que há o distanciamento presente nos discursos dos gerentes de saúde em relação ao preconizado pelas diretrizes do SUS. Nessa concepção, o discurso que ampara os sentidos para a realização do exame do TR anti-HIV em serviços de APS é predominado o discurso hegemônico, com peculiaridade biomédica e hospitalocêntrica, encaminhando os usuários para os serviços hospitalares especializados, além das barreiras existentes mencionadas em suas falas para obtenção do diagnóstico. 
Por meio dos discursos dos sujeitos desta pesquisa, pode-se depreender importantes discussões relacionadas à adequação da localização dos serviços em relação à residência dos usuários, ao atendimento integral, à qualidade e quantidade dos cuidados de saúde, aos aspectos relacionados ao transporte, além da percepção e atitudes dos profissionais de saúde a respeito das características pessoais dos usuários. Assim, estes achados possuem importante contribuição para a área da saúde pública em geral, tendo em vista a ampliação da compreensão acerca da problemática, apontando sentidos que otimizam a implementação das políticas públicas e auxiliam na tomada de decisão voltada à integralidade do acesso para a realização do TR anti-HIV na APS.

A realização do estudo restrita ao gerente de saúde pode ser apontada como possível limitação da pesquisa, tendo em vista que a inclusão de outros profissionais que exercem atividade direta com a oferta do TR anti-HIV poderiam ter contribuído para a melhor percepção do contexto avaliado. Analisar o discurso desses participantes é estratégico, pois permite assimilar o contexto das barreiras referentes à detecção do HIV na APS, visto que esses profissionais executam atividades administrativas com competência para suprimir estas barreiras, na perspectiva de qualificação de recursos humanos e na reestruturação do serviço de saúde.

\section{Considerações finais}

A efetivação da realização do TR anti-HIV na APS ainda se compreende como um problema para a gestão em saúde. Os discursos sinalizam o distanciamento da implantação das políticas de saúde e do comprometimento político, entrelaçados nas barreiras elencadas, tomando esses entraves como basilares na oferta do TR anti-HIV, o que é revelado por problemas quanto à infraestrutura inadequada, localização do serviço distante dos usuários, segurança local vulnerável, articulação fragilizada entre os pontos de atenção, horário de funcionamento centralizado, falta de familiaridade com a capacitação profissional, escassez de insumo, geração de custos evitáveis, discriminação de pessoas vivendo com HIV e estigmatização. Essa conjuntura requer a concepção e aplicação de políticas públicas de forma a auxiliar a gestão do cuidado ao HIV e reverter o quadro atual da infecção no país.

Os discursos possibilitaram identificar: a necessidade de capacitação/estratégias de educação permanente para os profissionais de saúde executarem, de maneira segura, o teste rápido, sem ter que direcionar a outro serviço ou solicitar o serviço de outras unidades; a responsabilidade por atender a demanda, sem a transferência para o serviço de referência; o provimento de insumos com fluxo contínuo de disponibilidade nas unidades; a organização para atender à demanda espontânea; a intersetorialidade para promover segurança no acesso, além do auxílio governamental à população socialmente vulnerável.

Assim, com base no material analisado, foi possível reconhecer informações que servirão de subsídios para propostas de novas metas e estratégias para potencializar a realização do TR, bem como sua utilização para produção de novos estudos que compreendam a ótica dos gestores saúde, além de outros profissionais e usuários.

\section{Colaborações:}

1 - concepção, projeto, análise e interpretação dos dados: Haline Costa dos Santos Guedes, José Nildo de Barros Silva Júnior, Amanda Haissa Barros Henriques e Anne Jaquelyne Roque Barrêto;

2 - redação do artigo e revisão crítica relevante do conteúdo intelectual: Haline Costa dos Santos Guedes, José Nildo de Barros Silva Júnior, Amanda Haissa Barros Henriques, Débora Raquel Soares Guedes Trigueiro, Jordana Almeida Nogueira e Anne Jaquelyne Roque Barrêto;

3 - aprovação final da versão a ser publicada: Anne Jaquelyne Roque Barrêto.

\section{Referências}

1. Raguin G, Girard P. Toward a global health approach: lessons from the HIV and Ebola 
epidemics. Glob Health. 2018;14(1):114. DOI: https://dx.doi.org/10.1186/s12992-018-0435-9

2. United Nations Programme on HIV/Aids. Estatísticas globais sobre HIV 2019 [Internet]. Brasília (DF); 2019 [cited 2020 Aug 12]. Available from: https://unaids.org.br/estatisticas

3. Brasil. Ministério da Saúde. Secretaria de Vigilância em Saúde. Departamento de Doenças de Condições Crônicas e Infecções Sexualmente Transmissíveis. Boletim Epidemiológico de HIV e Aids [Internet]. Brasília (DF); 2019 [cited 2020 Oct 22]. Available from: http://www.aids.gov. br/pt-br/pub/2019/boletim-epidemiologico-dehivaids-2019

4. Zambenedetti G, Silva RAN. Descentralização da atenção em HIV-Aids para a atenção básica: tensões e potencialidades. Physis. 2016;26(3):785-806. DOI: http://dx.doi.org/ 10.1590/S0103-73312016000300005

5. Melo EA, Maksud I, Agostini R. HIV/Aids management at the primary care level in Brazil: a challenge for the Unified Health System? Rev Panam Salud Publica. 2018;42:e151. DOI: https://dx.doi.org/10.26633/RPSP.2018.151

6. Frasca T, Fauré YA, Atlani-Duault L. Decentralisation of Brazil's HIV/AIDS programme: intended and unintended consequences. Glob Public Health. 2018;13(12):1725-36. DOI: https:// dx.doi.org/10.1080/17441692.2018.1455888

7. Silva ITS, Valença CN, Silva RAR. Mapping the implementation of the rapid HIV test in the Family Health Strategy: the nurses' perspective. Esc Anna Nery. 2017;21(4):e20170019. DOI: https://dx.doi.org/10.1590/2177-9465-ean2017-0019

8. Rocha KB, Ew RAS, Moro LM, Zanardo GLP, Pizzinato A. Counselling through the perspective of professionals of primary health care: challenges of decentralization of rapid test for HIV/Aids. Ciênc psicol. 2018;12(1):67-78. DOI: https://dx.doi.org/10.22235/cp.v12i1.1597

9. Silva YT, Silva LB, Ferreira SMS. Counseling practices in Sexually Transmitted Infections/AIDS: the female health professionals' perspective. Rev Bras Enferm. 2019;72(5):1137-44. DOI: https://dx.doi.org/10.1590/0034-7167-2018-0176

10. Guedes HCS, Silva Júnior JNB, Silva GNS, Trigueiro DRSG, Nogueira JA, Barrêto AJR. The integrality in Primary Healthcare: discourse analysis about the organization of the provision of the rapid anti-HIV test. Esc Anna Nery. 2021;25(1):e20190386. DOI: https://dx.doi. org/10.1590/2177-9465-EAN-2019-0386

11. Souza SAF. Análise de discurso: procedimentos metodológicos. Manaus: Census; 2014.

12. Jacobs B, Ir P, Bigdeli M, Annear PL, Van Damme W. Addressing access barriers to health services: an analytical framework for selecting appropriate interventions in low-income Asian countries. Health Policy Plan. 2012 July;27(4):288-300. DOI: https:// dx.doi.org/10.1093/heapol/czr038

13. Oliveira RAD, Duarte CMR, Pavão ALB, Viacava F. Barriers in access to services in five Health Regions of Brazil: perceptions of policymakers and professionals in the Brazilian Unified National Health System. Cad Saúde Pública. 2019;35(11):e00120718. DOI: https:// dx.doi.org/10.1590/0102-311X00120718

14. Zakabi D, Oliveira AFPL, Teixeira RR. Meetings and conversation during HIV testing in Primary Health Care in São Paulo. Essentia [Internet]. 2018 [cited 2020 Aug 23 2020];19(1):63-75. Available from: https://essentia. uvanet.br/index.php/ESSENTIA/article/view/164

15. Barreto HIV, Souza MKB. Analysis of health conditions of family health units based on the work process benchmark. Rev APS. 2017;20(4):519-26. DOI: https://doi.org/10.34019/ 1809-8363.2017.v20.15974

16. Paula CC, Silva CB, Tassinari TT, Padoin SMM. Factors that affect first contact access in the primary health care: integrative review. Rev pesqui cuid fundam online. 2016;8(1):4056-78. DOI: $10.9789 / 2175-5361.2016 . v 8 i 1.4056-4078$

17. Silva APF, Backes DS, Magnago TSBS, Colomé JS. Patient safety in primary care: conceptions of family health strategy nurse. Rev Gaúcha Enferm. 2019;40(esp):e20180164. DOI: https://dx.doi.org/10.1590/1983-1447.2019. 20180164

18. Araújo GM, Nardino LJ, Resdorfer N, Begnini D. Advise pre-testing: a proposal for a health education. Rev Esp Ciênc Saúde [Internet]. 2017 [cited 2020 Aug 24];5(1):61-73. Available: from: http://revistaeletronicaocs.unicruz.edu.br/ index.php/enfermagem/article/view/5493/1057

19. Vidal TB, Rocha SA, Harzheim E, Hauser L, Tesser CD. Scheduling models and primary health care quality. Rev Saúde Pública. 
2019;53(Suppl 1):38. DOI: https://dx.doi. org/10.11606/S1518-8787.2019053000940

20. Fertonani HP, Pires DEP, Biff D, Scherer MDA. The health care model: concepts and challenges for primary health care in Brazil. Ciênc saúde coletiva. 2015;20(6):1869-78. DOI: https://dx.doi. org/10.1590/1413-81232015206.13272014

21. Martins MMF, Aquino R, Pamponet ML, Pinto Junior EP, Amorim LDAF. Adolescent and youth access to primary health care services in a city in the state of Bahia, Brazil. Cad Saúde Pública. 2019;35(1):e00044718. DOI: https:// dx.doi.org/10.1590/0102-311X00044718

22. Magno L, Silva LAV, Veras MA, Pereira-Santos M, Dourado I. Stigma and discrimination related to gender identity and vulnerability to HIV/AIDS among transgender women: a systematic review. Cad Saúde Pública. 2019;35(4):e00112718. DOI: https://dx.doi.org/10.1590/0102-311X00112718

23. Stangl AL, Lilleston P, Mathema H, Pliakas T, Krishnaratne S, Sievwright K, et al. Development of parallel measures to assess HIV stigma and discrimination among people living with HIV, community members and health workers in the HPTN 071 (PopART) trial in Zambia and South Africa. J Int AIDS Soc. 2019;22:e25421. DOI: https:// dx.doi.org/10.1002/jia2.25421

24. Pantelic M, Casale M, Cluver L, Toska E, Moshabela M. Multiple forms of discrimination and internalized stigma compromise retention in HIV care among adolescents: findings from a South African cohort. J Int AIDS Soc. 2020;23(5):e25488. DOI: https://dx.doi.org/10. $1002 / \mathrm{jia} 2.25488$

25. Zambenedetti G, Silva RAN. Paradoxo do território e os processos de estigmatização no acesso ao diagnóstico de HIV na atenção básica em saúde. Estud psicol [Internet]. 2015 [cited 2020 Sep 15];20(4):229-40. Available from: https://www. lume.ufrgs.br/handle/10183/147185

Recebido: 23 de fevereiro de 2021

Aprovado: 28 de abril de 2021

Publicado: 2 de junho de 2021

A Revista Baiana de Enfermagem utiliza a Licença Creative Commons - Atribuição-NãoComercial 4.0 Internacional.

https://creativecommons.org/licenses/by-nc/4.0/

Este artigo é de acesso aberto distribuído sob os termos da Licença Creative Commons (CC BY-NC).

Esta licença permite que outros remixem, adaptem e criem a partir do seu trabalho para fins não comerciais. Embora os novos trabalhos tenham de lhe atribuir o devido crédito e não possam ser usados para fins comerciais, os usuários não têm de licenciar esses trabalhos derivados sob os mesmos termos. 